\title{
CENTRAL SEQUENCES ASSOCIATED WITH A STATE
}

\author{
SZE-KAI TSUI
}

\begin{abstract}
Central sequences associated with a state are defined and used to derive a characterization of the factor state in question. This characterization is used to study the factor state extension problem. One of the affirmative results obtained in this paper is as follows. Let $\mathscr{C}_{1}$ be a finite dimensional sub- $C^{*}$-algebra of $Q$. Then every factor state on the relative commutant of $Q_{1}$ in $Q$ extends to a factor state on $\mathbb{Q}$.
\end{abstract}

In this note a factor state on a $C^{*}$-algebra is characterized in terms of central sequences associated with it. The general concept of central sequences has been used in many cases to characterize different properties (see [1], [3], [4], and [5] for example). This characterization is then used to study factorial extensions of factor states from a sub- $C^{*}$-algebra to the $C^{*}$-algebra containing it.

1. A characterization of factor states. All $C^{*}$-algebras considered in this note are unital and separable. (In the general case, one may consider $\varphi$-central nets.) Let $\varphi$ be a state on $\mathbb{Q}$. For $y$ in $\mathbb{Q}$, let $\varphi \cdot y$ denote the element in $\mathbb{Q}^{*}$ (the dual of $\mathbb{Q}$ ) defined by $\varphi \cdot y(x)=\varphi(y x)$ for all $x \in \mathbb{Q}$, and $\mathbb{Q}^{+}=\{x \in \mathbb{Q} \mid x>0\}$.

1.1 Definition. A sequence of elements $\left\{x_{i} \in \mathbb{Q} \mid i=1,2, \ldots\right\}$ is said to be a $\varphi$-central sequence in $\mathbb{Q}$, if

$$
\lim _{i \rightarrow \infty}\left(\varphi \cdot y\left(x_{i} x\right)-\varphi \cdot y\left(x x_{i}\right)\right)=0 \text { for all } x, y \in \mathbb{Q} \text {. }
$$

A $\varphi$-central sequence $\left\{x_{i}\right\}$ is called convergent if $\varphi \cdot y\left(x_{i} x\right)$ converges for all $x, y \in \mathbb{Q}$, and is called positive if $0<x_{i}$ for all $i$. A positive $\varphi$-central sequence $\left\{x_{i}\right\}_{n=1}^{\infty}$ is $k$-bounded, if, for all $i, \sup _{\varphi\left(y^{*} y\right)<1} \varphi\left(y^{*} x_{i} y\right)<k$. Two $\varphi$-central sequences $\left\{x_{i}\right\}$ and $\left\{z_{i}\right\}$ in $\mathcal{Q}$ are said to be equivalent, if $\lim _{i \rightarrow \infty}\left(\varphi \cdot y\left(x_{i}\right)-\varphi \cdot y\left(z_{i}\right)\right)$ $=0$ for all $y$ in $\mathcal{Q}$. A $\varphi$-central sequence $\left\{x_{i}\right\}$ is trivial, if there exists a sequence of complex numbers $\left\{\lambda_{i}\right\}$ which is equivalent to $\left\{x_{i}\right\}$.

In the following we tie the existence of a nontrivial $\varphi$-central sequence to the fact that $\varphi$ is not a factor state. Let $\pi_{\varphi}, \mathcal{H}_{\varphi}$ be the GNS representation and its representation space induced by $\varphi$, and $\varphi\left(y^{*} x\right)=\left\langle\pi_{\varphi}(x) f_{\varphi}, \pi_{\varphi}(y) f_{\varphi}\right\rangle$ for all $x, y$ in $\mathscr{Q}$. Let $\mathscr{R}=\pi_{\varphi}(\mathscr{Q})^{\prime \prime}$ and $\mathscr{Z}(\Re)$ be the center of $\Re$. This notation is used for the rest of the paper.

1.2 Proposition. Every $A \in \mathscr{Z}^{+}(\Re)$ with $\|A\|<1$ gives rise to a positive 1-bounded convergent $\varphi$-central sequence $\left\{x_{i}\right\}$ in $\mathbb{Q}$ such that $\left\langle A \pi_{\varphi}(z) f_{\varphi}, \pi_{\varphi}(y) f_{\varphi}\right\rangle=$ $\lim _{i \rightarrow \infty} \varphi\left(y^{*} x_{i} z\right)$ for all $z, y$ in $\mathbb{Q}$.

Received by the editors March 16, 1980; presented to the Society, January 4, 1980.

1980 Mathematics Subject Classification. Primary 46L30, 46LO5.

Key words and phrases. Central sequences associated with a state, factor states, Kaplansky's density theorem, factor state extension, extreme points, projection mapping of norm one. 
Proof. Since $A$ is in $\mathscr{Z}^{+}(\Re)$, by Kaplansky's density theorem there exists a sequence of positive elements $\left\{x_{i}\right\}$ in $\mathcal{Q}$ with $\left\|\pi_{\varphi}\left(x_{i}\right)\right\|<\|A\|$ such that $\left\{\pi_{\varphi}\left(x_{i}\right)\right\}$ converges to $A$ in the ultraweak operator topology, and $\left\{x_{i}\right\}$ is 1-bounded. In particular, $\lim _{i \rightarrow \infty}\left\langle\left(\pi_{\varphi}\left(x_{i}\right)-A\right) \pi_{\varphi}(x) f_{\varphi}, \pi_{\varphi}(y) f_{\varphi}\right\rangle=0$ for all $x, y$ in $\mathbb{Q}$. That is

$$
\lim _{i \rightarrow \infty} \varphi\left(y x_{i} z\right)=\left\langle A \pi_{\varphi}(z) f_{\varphi}, \pi_{\varphi}\left(y^{*}\right) f_{\varphi}\right\rangle \text { for all } z, y \in \mathbb{Q} \text {. }
$$

Replacing $y$ by $y x$ and $z$ by 1 in (2), we derive

$$
\begin{aligned}
\lim _{i \rightarrow \infty} \varphi\left(y x x_{i}\right) & =\left\langle A f_{\varphi}, \pi_{\varphi}\left(x^{*}\right) \pi_{\varphi}\left(y^{*}\right) f_{\varphi}\right\rangle \\
& =\left\langle A \pi_{\varphi}(x) f_{\varphi}, \pi_{\varphi}\left(y^{*}\right) f y\right\rangle \\
& =\lim _{i \rightarrow \infty} \varphi\left(y x_{i} x\right) .
\end{aligned}
$$

Hence $\lim _{i \rightarrow \infty} \varphi \cdot y\left(x_{i} x\right)$ exists, and

$$
\lim _{i \rightarrow \infty}\left[\varphi \cdot y\left(x_{i} x\right)-\varphi \cdot y\left(x x_{i}\right)\right]=0 \quad \text { for all } x, y \in \mathbb{Q} \text {. Q.E.D. }
$$

The $\varphi$-central sequence in Proposition 1.2 is called a $\varphi$-central sequence determined by $A$. In the remainder of this section we are going to establish a one-to-one correspondence between $\mathscr{Z}^{+}(\mathscr{R})$ and the collection of all equivalence classes of positive bounded convergent central sequences in $\mathbb{Q}$.

1.3. Proposition. Given a positive $k$-bounded convergent $\varphi$-central sequence $\left\{x_{i}\right\}$, there exists an element $A$ in $\mathscr{Z}^{+}(\Re)$ which determines the equivalence class of $\left\{x_{i}\right\}$. Furthermore $\|A\| \leqslant k$.

Proof. Let $\left\{x_{i}\right\}$ be a positive convergent $k$-bounded $\varphi$-central sequence in $\mathbb{Q}$ with $k<\infty$. Denote $\lim _{i \rightarrow \infty} \varphi \cdot y\left(x_{i} x\right)=\psi(y, x)$. It is easily seen that

(i) $\psi$ is bilinear on $\mathbb{Q} \times \mathbb{Q}$.

(ii) $\psi\left(y^{*}, y\right)=\lim _{i \rightarrow \infty} \varphi\left(y^{*} x_{i} y\right)>0$ and $\psi\left(y^{*}, y\right)=\lim _{i \rightarrow \infty} \varphi\left(y^{*} x_{i} y\right)<$ $k \varphi\left(y^{*} y\right)$.

Define a positive semidefinite sesquilinear functional on $H_{\varphi} \times H_{\varphi}$ by

$$
\phi\left(\pi_{\varphi}(x) f_{\varphi}, \pi_{\varphi}(y) f_{\varphi}\right)=\psi\left(y^{*}, x\right) \text { for } x, y \in \mathbb{Q} .
$$

It is also bicontinuous. In fact,

$$
\begin{aligned}
\left|\phi\left(\pi_{\varphi}(x) f_{\varphi}, \pi_{\varphi}(y) f_{\varphi}\right)\right| & =\lim _{i \rightarrow \infty}\left|\varphi\left(y^{*} x_{i} x\right)\right|<\lim _{i \rightarrow \infty} \varphi\left(y^{*} x_{i} y\right)^{1 / 2} \varphi\left(x x_{i} x\right)^{1 / 2} \\
& \leqslant \lim _{i \rightarrow \infty} k \varphi\left(y^{*} y\right)^{1 / 2} \varphi\left(x^{*} x\right)^{1 / 2}=k\left\|\pi_{\varphi}(x) f_{\varphi}\right\|\left\|\pi_{\varphi}(y) f_{\varphi}\right\| .
\end{aligned}
$$

By Riesz's representation theorem [9, p. 90], there exists an operator $A$ in $B\left(\mathcal{H}_{\varphi}\right)$ such that

$$
\begin{aligned}
\left\langle A \pi_{\varphi}(x) f_{\varphi}, \pi_{\varphi}(y) f_{\varphi}\right\rangle & =\psi\left(y^{*}, x\right)=\lim _{i \rightarrow \infty} \varphi\left(y^{*} x_{i} x\right) \\
& =\lim _{i \rightarrow \infty}\left\langle\pi_{\varphi}\left(x_{i}\right) \pi_{\varphi}(x) f_{\varphi}, \pi_{\varphi}(y) f_{\varphi}\right\rangle .
\end{aligned}
$$

Hence $\pi_{\varphi}\left(x_{i}\right)$ converges to $A$ in weak operator topology, and $A \in R$. It is also clear that $A$ is positive and $\|A\|<k$. 
Since $\psi(y, x)=\lim _{i \rightarrow \infty} \varphi \cdot y\left(x_{i} x\right)=\lim _{i \rightarrow \infty} \varphi \cdot y\left(x x_{i}\right)=\lim _{i \rightarrow \infty} \varphi\left(y x x_{i}\right)=$ $\psi(y x, 1),(x, y \in \mathbb{Q})$, we have

$$
\psi\left(y^{*}, x z\right)=\psi\left(y^{*} x z, 1\right)=\psi\left(y^{*} x, z\right)=\psi\left(\left(x^{*} y\right)^{*}, z\right),
$$

which is the same as

$$
\begin{aligned}
\left\langle A \pi_{\varphi}(x) \pi_{\varphi}(z) f_{\varphi}, \pi_{\varphi}(y) f_{\varphi}\right\rangle & =\left\langle A \pi_{\varphi}(z) f_{\varphi}, \pi_{\varphi}(x)^{*} \pi_{\varphi}(y) f_{\varphi}\right\rangle \\
& =\left\langle\pi_{\varphi}(x) A \pi_{\varphi}(z) f_{\varphi}, \pi_{\varphi}(y) f_{\varphi}\right\rangle
\end{aligned}
$$

for all $x, y, z$ in $\mathscr{Q}$. Hence $A \pi_{\varphi}(x)=\pi_{\varphi}(x) A,(x \in \mathbb{Q})$, and $A \in \mathscr{Z}^{+}(\Re)$. Q.E.D.

The above proof is analogous to the proof of the theorem that pure states are those states inducing an irreducible representation in GNS construction [7].

1.4. THEOREM. $\varphi$ is a factor state if and only if all bounded positive convergent $\varphi$-central sequences are equivalent to trivial sequences.

Proof. It follows evidently from 1.2 and 1.3. Q.E.D.

2. Extension of factor states. The usefulness of the above characterization of factor states can be illustrated in the study of the possibility for factorial extensions of a factor state from a sub- $C^{*}$-algebra to the $C^{*}$-algebra containing it.

Let $\mathscr{B}$ be a sub- $C^{*}$-algebra of a $C^{*}$-algebra $\mathcal{Q}$ and $\varphi$ be a state on $\mathscr{B}$. The state space of $\mathscr{Q}$ is denoted by $\mathcal{S}(\mathscr{Q})$ and $\mathscr{K}_{\varphi}=\left\{\psi \in \mathcal{S}(\mathbb{Q})|\psi|_{\mathscr{B}}=\varphi\right\} . \mathcal{K}_{\varphi}$ is a weak* compact convex subset of $\mathscr{Q}^{*}$, and it is always nonempty [2, 2.10.1]. By the Krein-Milman Theorem we can assume the existence of an extreme point, $\psi_{0}$, in $\mathcal{K}_{\varphi}$.

2.1. Definition. Let $\left\{x_{i}\right\}$ be a $\varphi$-central sequence in $\mathcal{Q}$ and $\left\{y_{i}\right\}$ be a $\varphi$-central sequence in $\mathscr{B} .\left\{y_{i}\right\}$ is called a restriction of $\left\{x_{i}\right\}$ in $\mathscr{B}$ if

$$
\lim _{i \rightarrow \infty}\left[\varphi \cdot y\left(x_{i}\right)-\varphi \cdot y\left(y_{i}\right)\right]=0 \text { for all } y \text { in } \mathscr{B} \text {. }
$$

2.2. Proposition. Suppose $\varphi$ is a factor state. $\psi_{0}$, an extreme point in $\mathcal{K}_{\varphi}$, is a factor state if every bounded positive $\psi_{0}$-central sequence in $\mathbb{Q}$ has a restriction in $\mathscr{B}$ which is bounded positive and convergent.

Proof. Let $\left\{\pi_{\psi_{0}}, \mathcal{K}_{\psi_{0}}\right\}$ be the GNS representation induced by $\psi_{0}$ and $f_{\psi_{0}}$ be the cyclic vector of $\pi_{\psi_{0}}(Q)$. Without loss of generality we may let $\left\{x_{i}\right\}$ be a 1-bounded positive convergent $\psi_{0}$-central sequence in $\mathcal{Q}$, with $\lim _{i \rightarrow \infty} \psi_{0}\left(x_{i}\right)=\lambda>0$, and $A$ be the element in $\mathscr{Z}^{+}\left(\pi_{\psi_{0}}(\mathscr{Q})^{\prime \prime}\right)$ determining $\left\{x_{i}\right\}$ by Propositions 1.2 and 1.3. By the assumption, there exists a bounded positive convergent $\varphi$-central sequence $\left\{z_{i} \mid z_{i} \in\right.$ $\mathscr{B}\}$ that is a restriction of $\left\{x_{i}\right\}$ in $\mathscr{B}$. Since $\varphi$ is a factor state on $\mathscr{B}$, it follows from Theorem 1.4 that $\left\{z_{i}\right\}$ is equivalent to a trivial sequence $\left\{\lambda_{i}\right\}$ with $\lim _{i \rightarrow \infty} \varphi\left(z_{i}\right)=$ $\lim _{i \rightarrow \infty} \lambda_{i}=\lambda \leqslant 1$.

Consider a positive linear functional on $\mathcal{Q}$ defined by $\psi_{A}(x)=\left\langle A \pi_{\psi_{0}}(x) f_{\psi_{0}}, f_{\psi_{0}}\right\rangle$ for $x$ in $Q$. It is clear that $\psi_{A}<\psi_{0}$. For $z$ in $\mathscr{B}$ we have

$$
\begin{aligned}
\psi_{A}(z) & =\lim _{i \rightarrow \infty} \psi_{0}\left(x_{i} z\right)=\lim _{i \rightarrow \infty} \psi_{0}\left(z x_{i}\right)=\lim _{i \rightarrow \infty} \psi_{0}\left(z z_{i}\right) \\
& =\lim _{i \rightarrow \infty} \psi_{0}\left(z \lambda_{i}\right)=\lambda \psi_{0}(z)=\lambda \varphi(z) .
\end{aligned}
$$


Hence $\psi_{A} / \lambda$ is a state which extends $\varphi$. If $\lambda=1$, it follows from $\psi_{0}-\psi_{A}>0$ and $\left(\psi_{0}-\psi_{A}\right)(I)=0$ that $\left\|\psi_{0}-\psi_{A}\right\|=0$. Hence $\psi_{0}=\psi_{A}$, and $A=I$. If $\lambda<1$, then

$$
\psi_{0}=\lambda\left(\frac{1}{\lambda} \psi_{A}\right)+(1-\lambda)\left(\frac{1}{1-\lambda}\left\{\psi_{0}-\psi_{A}\right\}\right) \text {. }
$$

Thus $\psi_{0}=\psi_{A} / \lambda$ because $\psi_{0}$ is extremal in $\mathcal{K}_{\varphi}$. This implies that $\lim _{i \rightarrow \infty} \psi_{0}\left(x x_{i}\right)=$ $\lambda \psi_{0}(x)$ for all $x$ in $\mathcal{Q}$, namely, $\left\{x_{i}\right\}$ is equivalent to a trivial sequence $\left\{\lambda_{i} \mid \lambda_{i}=\lambda\right\}$ in $Q$. Therefore, by Theorem 1.4, $\psi_{0}$ is a factor state. Q.E.D.

In what follows, let $M_{n}$ be the $n \times n$ full matrix algebra (over the complex field). Suppose that $M_{n}$ is a sub-C*-algebra of $C^{*}$-algebra $\mathcal{Q}$, and let $\mathscr{B}=M_{n}^{c}$, the commutant of $M_{n}$ in $\mathscr{Q}$ (i.e., $\mathscr{B}=\left\{x \in \mathcal{Q} \mid x y=y x\right.$ for all $y$ in $\left.M_{n}\right\}$ ).

2.3. TheOREM. Every factor state $\varphi$ of $\mathscr{B}$ extends to a factor state on $\mathbb{Q}$.

Proof. Let $\psi_{0}$ be an extreme point in $\mathscr{K}_{\varphi},\left\{x_{i}\right\}$ be a bounded positive convergent $\psi_{0}$-central sequence, and $A$ be the element in $\mathscr{Z}\left(\pi_{\psi_{0}}(\mathscr{Q})^{\prime \prime}\right)$ determining $\left\{x_{i}\right\}$. We denote the matrix units of $M_{n}$ by $\left\{e_{r s} \mid r, s=1, \ldots, n\right\}$.

For any $x, y$ in $\mathscr{B}$ and $r$ with $1 \leqslant r \leqslant n$ we have

$$
\lim _{i \rightarrow \infty} \psi_{0} \cdot y\left(e_{r 1} x_{i} e_{1 r} x\right)=\lim _{i \rightarrow \infty} \psi_{0} \cdot y\left(x e_{r 1} x_{i} e_{1 r}\right)
$$

Letting $\hat{x}_{i}=\sum_{r=1}^{n} e_{r 1} x_{i} e_{1 r}, i=1,2, \ldots$, we derive from (3) that, for any $x, y$ in $\mathscr{B}$,

$$
\lim _{i \rightarrow \infty} \psi_{0} \cdot y\left(x \hat{x}_{i}\right)=\lim _{i \rightarrow \infty} \psi_{0} \cdot y\left(\hat{x}_{i} x\right)
$$

On the other hand, since $A$ determines $\left\{x_{i}\right\}$, by Proposition 1.2 we have, for $x, y$ in $\mathscr{B}$ and $r=1,2, \ldots, n$,

$$
\begin{aligned}
\lim _{i \rightarrow \infty} \psi_{0} \cdot y\left(e_{r 1} x_{i} e_{1 r} x\right) & =\left\langle A \pi_{\psi_{0}}\left(e_{1 r} x\right) f_{\psi_{0}}, \pi_{\psi_{0}}\left(e_{1 r} y^{*}\right) f_{\psi_{0}}\right\rangle \\
& =\left\langle A \pi_{\psi_{0}}\left(e_{r 1} e_{1 r} x\right) f_{\psi_{0}}, \pi_{\psi_{0}}\left(y^{*}\right) f_{\psi_{0}}\right\rangle \\
& =\left\langle A \pi_{\psi_{0}}\left(e_{r r} x\right) f_{\psi_{0}}, \pi_{\psi_{0}}\left(y^{*}\right) f_{\psi_{0}}\right\rangle .
\end{aligned}
$$

It follows from (5) that, for $x, y$ in $\mathscr{B}$, we have

$$
\lim _{i \rightarrow \infty} \psi_{0} \cdot y\left(\hat{x}_{i} x\right)=\left\langle A \pi_{\psi_{0}}(x) f_{\psi_{0}}, \pi_{\psi_{0}}\left(y^{*}\right) f_{\psi_{0}}\right\rangle=\lim _{i \rightarrow \infty} \psi_{0} \cdot y\left(x_{i} x\right) .
$$

It is easy to see that $\hat{x}_{i}$ 's are in $M_{n}^{c}=\mathscr{B}$. It follows from (4), (6) and $\left\|\pi_{\psi_{0}}\left(\hat{x}_{i}\right)\right\|<$ $\left\|\pi_{\psi_{0}}\left(x_{i}\right)\right\|$ that $\left\{\hat{x}_{i}\right\}$ is a bounded positive convergent $\psi_{0}$-central sequence in $\mathscr{B}$ which is a restriction of $\left\{x_{i}\right\}$. By Proposition $2.2 \psi_{0}$ is a factor state. Q.E.D.

2.4. Remark. Let $\mathcal{Q}_{1}$ be a finite dimensional sub- $C^{*}$-algebra of $\mathcal{Q}$, namely, $\mathcal{Q}_{1}=\sum_{j=1}^{k} \bigoplus M_{n_{j}}$. Denote the commutant of $\mathcal{Q}_{1}$ in $\mathcal{Q}$ by $\mathscr{B}$. The proof of Theorem 2.3 can be slightly generalized to show that every factor state on $\mathscr{B}$ extends to a factor state on $\mathcal{Q}$. In fact, redefining $\hat{x}_{i}$ in the proof of Theorem 2.3 by

$$
\hat{x}_{i}=\sum_{j=1}^{k} \sum_{r=1}^{n_{j}} e_{r 1}^{j} e_{1 r}^{j},
$$

where $\left\{e_{r h}^{j}\right\}_{r, h=1}^{n_{j}}$ are the matrix units of $M_{n}$, and replacing the $e_{r 1}$ in conditions (3) and (5) of the proof of Theorem 2.3 by $e_{r 1}^{j}$ for $r=1,2, \ldots, n_{j}$ and $j=1, \ldots, k$, we can prove the above assertion by the same argument as in Theorem 2.3. 
2.5. REMARK. Let $P$ be a projection mapping of $\mathscr{Q}$ onto $\mathscr{B}$ of norm one. Due to [8, §3], $P$ is always completely positive. Suppose that $\varphi$ is a factor state on $\mathscr{B}$, and $\psi_{0}$ is an extreme point in $\mathcal{K}_{\varphi}$ such that $\psi_{0}=\varphi \circ P$. For any bounded positive convergent $\psi_{0}$-central sequence $\left\{x_{n}\right\}$ in $\mathcal{Q}$, we have

$$
\lim _{n \rightarrow \infty}\left|\psi_{0}\left(y x_{n} x\right)-\psi_{0}\left(y x x_{n}\right)\right|=0 \text { for } x, y \text { in } \mathcal{Q} .
$$

In particular, if $x, y$ are in $\mathscr{B}$ we have

$$
\left\{\begin{array}{l}
\lim _{n \rightarrow \infty}\left|\varphi\left(y P\left(x_{n}\right) x\right)-\varphi\left(y x P\left(x_{n}\right)\right)\right|=0, \\
\lim _{n \rightarrow \infty}\left|\varphi \circ P\left(y x_{n}\right)-\varphi \circ P\left(y P\left(x_{n}\right)\right)\right|=0 .
\end{array}\right.
$$

It follows from (7) that $\left\{P\left(x_{n}\right)\right\}$ is a bounded positive convergent $\psi_{0}$-central sequence in $\mathscr{B}$ which is a restriction of $\left\{x_{n}\right\}$ in $\mathscr{B}$. By Proposition $2.2 \psi_{0}$ is a factor state. If one considers $\mathcal{S}_{\varphi}=\{\varphi \circ P \mid P=$ projection mapping of $A$ onto $\mathscr{B}$ of norm one\}, it would be interesting to determine if the extreme points in $\delta_{\varphi}$, when they exist, are factor states on $\mathbb{Q}$.

\section{REFERENCES}

1. Charles Akemann and Gert Pedersen, Central sequences and inner derivations of separable $C^{*}$-algebras, preprint.

2. Jacques Dixmier, Les $C^{*}$-algèbres et leurs representations, Gauthier-Villars, Paris, 1969.

3. George Elliott, Some $C^{*}$-algebras with outer derivations. III, Ann. of Math. (2) 106 (1977), 121-143.

4. Dusa McDuff, Central sequences and the hyperfinite factor, Proc. London Math. Soc. (3) 21 (1970), 443-461.

5. Shôichirô Sakai, On automorphism groups of $\mathrm{II}_{1}$-factors, Tôhoku Math. J. (2) 26 (1974), 423-430.

6. $C^{*}$-algebras and $W^{*}$-algebras, Springer-Verlag, Berlin and New York, 1971.

7. I. E. Segal, Irreducible representations of operator algebras, Bull. Amer. Math. Soc. 53 (1947), 73-88.

8. Jun Tomiyama, Tensor products and projections of norm one in von Neumann algebras, Seminar at Univ. of Copenhagen, 1970.

9. Kôsaku Yosida, Functional analysis, 2nd ed., Die Grundlehren der Math. Wissenschaften, Bd. 123, Springer-Verlag, Berlin and New York, 1968.

Department of Mathematical Sciences, Oakiand Universtty, Rochester, Michigan 48063 